\title{
Human Joint Angle Estimation with Multiple-strip E-textile Stretching Sensor
}

\author{
Sang-Ho Han, ${ }^{1}$ Mun-Ho Ryu, ${ }^{2,3 *}$ and Je-Nam Kim ${ }^{4}$ \\ ${ }^{1}$ Department of Healthcare Engineering, Chonbuk National University, \\ 567 Baekje-daero, Deokjin, Jeonju, Jeollabuk 561-756, Republic of Korea \\ ${ }^{2}$ Division of Biomedical Engineering, Chonbuk National University, \\ 567 Baekje-daero, Deokjin, Jeonju, Jeollabuk 561-756, Republic of Korea \\ ${ }^{3}$ Research Center of Healthcare \& Welfare Instrument for the Aged, \\ 567 Baekje-daero, Deokjin, Jeonju, Jeollabuk 561-756, Republic of Korea \\ ${ }^{4}$ CAMTIC Advanced Mechatronics Technology Institute for Commercialization, \\ 67 Yu-Sang-ro, Deokjin, Jeonju, Jeollabuk 561-844, Republic of Korea
}

(Received April 5, 2017; accepted January 23, 2018)

Keywords: elbow angle, e-textile, stretch sensor, biomechanics, motion tracking

There have been many studies on strain-resistance or piezoresistance e-textile sensors. However, the important and critical factor, i.e., the elongation capability of the sensor against the actual stretching of the skin, has not been addressed yet. In this study, we propose a human joint angle estimation method with e-textile strain-resistance sensors. The stretching of the skin, on which the sensor is attached, is visually analyzed in terms of the stretching capability of the sensor and the actual stretching length of the skin. The overall system platform used to obtain and process the sensor data is described. The proposed system is implemented and applied to the elbow joint of a healthy subject. It was found that the sensors should be attached to the skin properly, especially when the skin is not stretched. It is necessary to consider the stretching capability of the sensor to accommodate the actual stretching of the skin. The two sensors attached on the lateral and medial sides provide complementary information, which can be utilized in a sensor fusion context.

\section{Introduction}

Electric textile (e-textile) sensors have recently attracted considerable attention in various research fields, including in human body measurement. ${ }^{(1-8)}$ E-textile sensors provide electrical characteristics such as resistance or capacitance according to the applied physical properties. With their adaptability to different body shapes, e-textile sensors provide greater comfort and flexibility to the wearer, compared with conventional rigid sensors. They also have benefits such as low cost and weight, and the ability to be integrated with clothing.

There have been many studies on strain-resistance or piezoresistance e-textile sensors. $\mathrm{Xu}$ et al. reported a sitting-posture monitoring system, ${ }^{(3)}$ for which they applied an array of piezoresistance sensors to a cushion, and classified the sitting postures into several classes. 
Mattmann et al. applied a strain sensor to recognize various upper-body postures. ${ }^{(4)}$ Shyr et al. developed an elastic conductive webbing and applied it to a wearable gesture-sensing device. ${ }^{(5)}$ The De Rossi group performed several intensive studies on human joint measurement with e-textile sensors. ${ }^{(6,7)}$ Their electronic goniometer with a double-layered configuration shows promising performance even for stretching properties. However, when a stretch sensor is applied to the human body, the elongation capability of the sensor against the actual stretching of the skin is an important and critical factor. Nevertheless, so far, most of the studies have not adequately considered this issue.

In this study, we propose a human joint angle estimation method with e-textile stainresistance sensors, i.e., stretching sensors. The stretching shape of the skin, on which the sensor is attached, is visually analyzed in terms of the stretching capability of the sensor and the actual stretching length of the skin. This is the main aim of this study. The configuration of the sensor array and the attachment location are designed considering the stretching shape of the skin. In addition, the overall system platform used to obtain and process the sensor data is described. The proposed system is implemented and applied to a healthy subject's elbow joint.

\section{Materials and Methods}

\subsection{Sensor data acquisition system}

The sensor data acquisition system consists of a PC, a microcontroller unit (MCU; Arduino Due, arduino.cc), constant current sources, stretching sensors (EeonTex ${ }^{\mathrm{TM}}$ LLT-SLPA, Eeonyx, CA), and buffers [Fig. 1(a)]. The sensor is made of a bidirectionally stretchy, knitted nylon $(72 \%)$ spandex (28\%) material coated with a proprietary conductive formation. ${ }^{(8)}$ The resistance of the sensor decreases as its length increases. In this study, the sensor was cut into a single piece with a length of $100 \mathrm{~mm}$ and a width of $20 \mathrm{~mm}$, and had a resistance of about $200 \mathrm{k} \Omega$ in the relaxed state. It was found that the length of $100 \mathrm{~mm}$ could stretch up to $120 \mathrm{~mm}$ with acceptable sensitivity and strain (20\% strain limit) ranges. The current sources provide constant current to induce a voltage drop. The voltage across the sensor strip is measured by the analog-

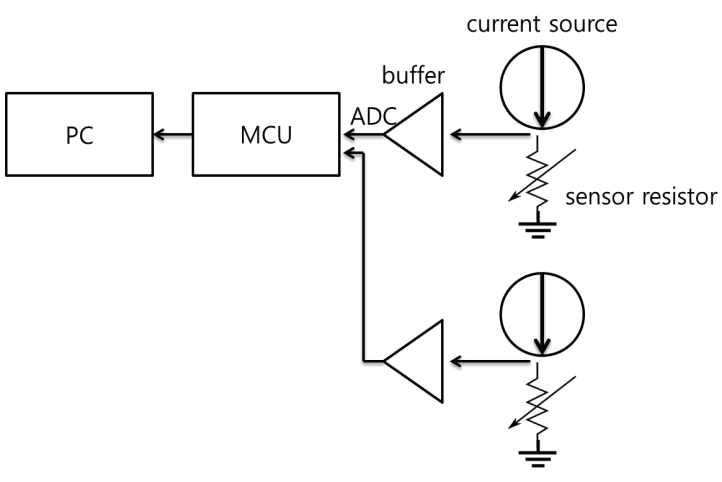

(a)

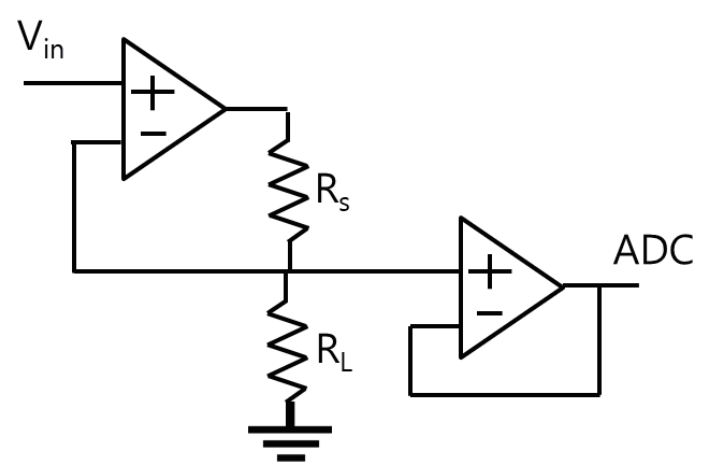

(b)

Fig. 1. (a) Sensor data acquisition system and (b) constant current source and buffer circuit. 
to-digital-converters (ADCs) in the MCU, and converted into resistance using Ohm's law. Since the resistance of the sensor is too high to be sampled and tracked by the ADC, its resistance is lowered using a buffer (LM385, Linear Technology, CA).

The constant current source is implemented as a voltage-to-current converter with an operational amplifier (LM385, Linear Technology, CA) [Fig. 1(b)]. The source current is adjusted with the input voltage $V_{i n}$ and the negative feedback resistor $R_{S}$ (based on Ohm's law). To obtain different currents, different voltages across the load resistor $R_{L}$, i.e., the sensor resistor, were measured and converted to the corresponding current. The actual measured current was scaled with the targeted current. The nonlinearity of the actual current along the various resistance values was calibrated by curve fitting with a third-order polynomial equation.

\subsection{Stretching shape analysis}

As mentioned earlier, when a stretch sensor is attached to the human body, the e-textile sensor stretches with the skin. If the sensor does not sufficiently elongate or does not maintain its sensitivity, the sensor loses its functionality. This is also true when the clothing or assisting textile, on which the sensor is attached, does not provide sufficient elongation even though the sensor itself provides the necessary elongation.

To model this situation, the stretching shape of the skin was analyzed with a stretchable arm sleeve. Before the arm sleeve was worn, $15 \times 15$ grids were marked with $5 \mathrm{~mm}$ intervals. Then, after the arm sleeve was worn over the elbow and fully flexed, the distances between the grid markers were measured with a ruler [Fig. 2(a)].

The measured distances were accumulated to obtain the total stretching distances along the lateral and proximal directions [Fig. 2(b)]. For instance, the original $70 \mathrm{~mm}$ distance of the

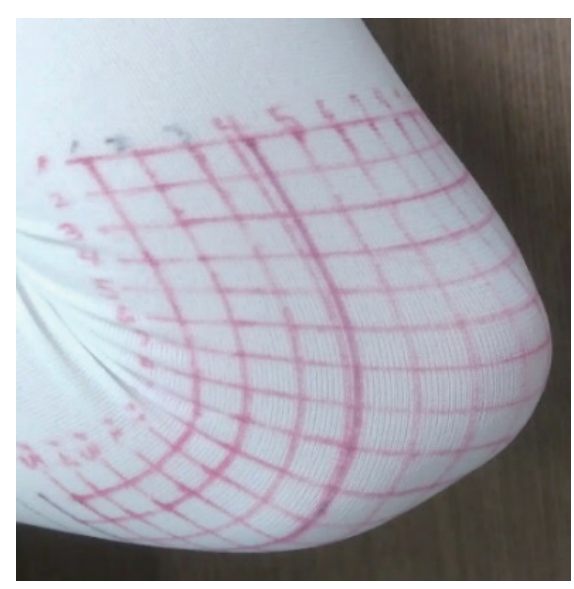

(a)

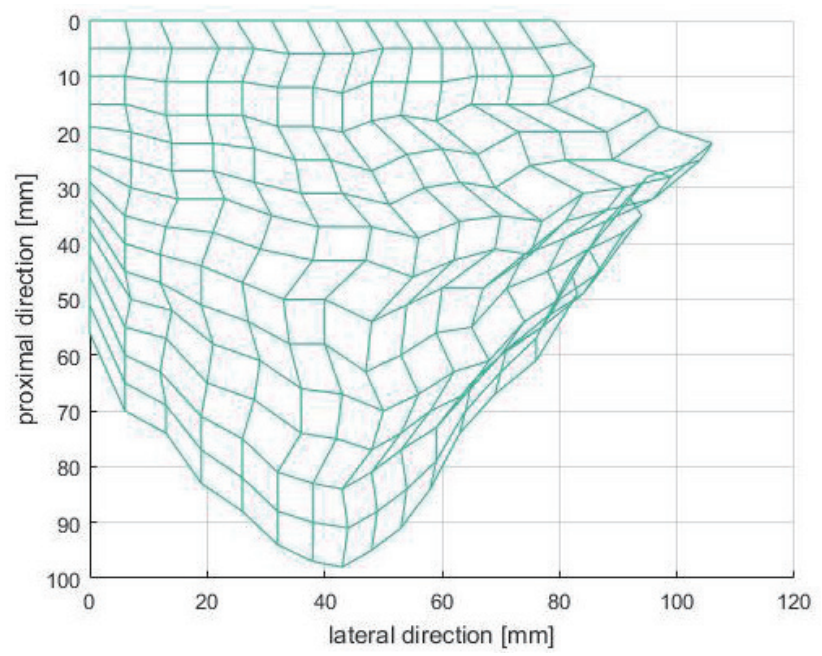

(b)

Fig. 2. (Color online) (a) Arm sleeve worn and fully flexed and (b) accumulated stretching distances. 
14 grids along the proximal direction via the coronoid process was stretched to $100 \mathrm{~mm}$. This stretching corresponds to $43 \%$ strain, and therefore the sensor in this study cannot be used, since it is only capable of a maximum strain of $20 \%$. Another interesting region is the lateral side of the elbow. In this region, the grids were shortened rather than elongated, and the sensor may not provide adequate functionality.

Besides the total stretching mentioned above, the individual stretching of the grid, i.e., local stretching, also appears to be important. For this case, the diagonal lengths of each stretched grid were calculated and plotted in 2D and 3D contours (Fig. 3). Any grid region with more than $20 \%$ strain, i.e., $8.5 \mathrm{~mm}$, should be avoided.

\section{Experimental Protocol}

The stretching characteristics of the sensor were investigated under both static and dynamic conditions. For this test, the sensor was stretched with a linear actuator (L12, Firgelli Technologies, Canada). Another Arduino Due MCU controlled the position of the actuator with a pulse-width modulation interface. The sensor textile was cut into a $120 \times 20 \mathrm{~mm}^{2}$ strip. Two points, which were $100 \mathrm{~mm}$ apart and within $10 \mathrm{~mm}$ of each end of the strip, were connected to the sensor data acquisition system described earlier. One end of the sensor was fixed with a clamp, and the other end was pulled for the elongation and relaxed for the shortening. The resistance values were sampled at $100 \mathrm{~Hz}$ and low-pass filtered below $0.5 \mathrm{~Hz}$ with a third-order Butterworth IIR filter.

For the static stretching test, the sensor was elongated from its resting position of 0 to $28 \mathrm{~mm}$ in $2 \mathrm{~mm}$ steps, and relaxed in the reverse order. For each stretching state, 500 samples (over $5 \mathrm{~s}$ ) of resistance were sampled and averaged. The elongation and shortening sequence was repeated five times. For the dynamic stretching test, the sensor was stretched from the $100 \mathrm{~mm}$ position to the $120 \mathrm{~mm}$ position. During this stretching, the measured resistance values were sampled, and the values before and after the stretching were compared. The nonfiltered resistance values were also sampled and compared.

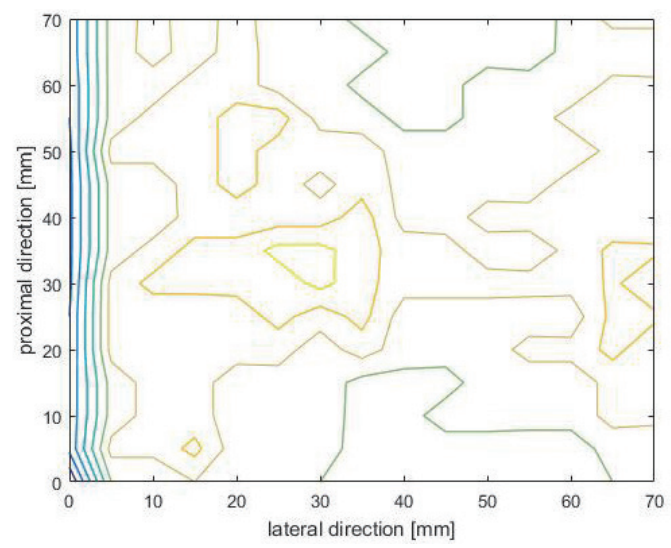

(a)

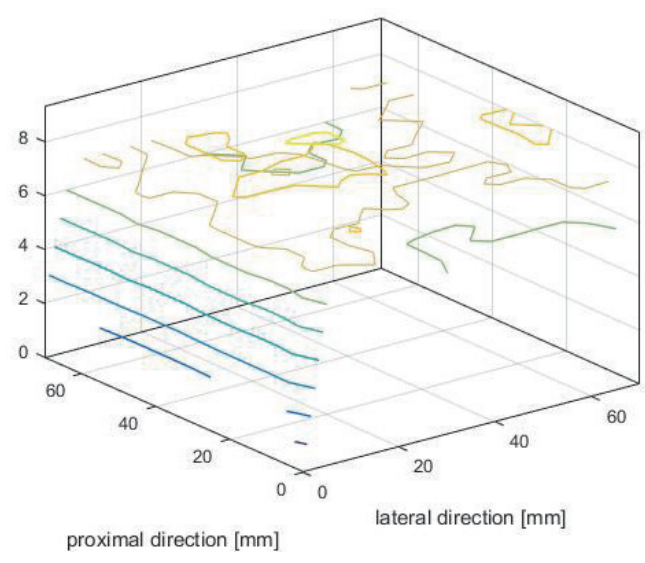

(b)

Fig. 3. (Color online) Local stretching distances in (a) 2D contour and (b) 3D contour. 
Two stretching sensors were attached on the elbow of a healthy subject via a stretchable arm sleeve. Each sensor was prepared in the same manner as in the stretching test. It was attached on a single-adhesive Kinesio tape that has $10 \mathrm{~mm}$ margins on four sides for attachment. The adhesive side of the Kinesio tape was attached to the sleeve. The sensors were attached on the lateral and medial sides along the proximal-distal direction, avoiding the coronoid process (Fig. 4). The resistance of the pair of sensors was measured from 0 to $120^{\circ}$ at $10^{\circ}$ intervals during flexion and extension, 6 times. During the test, the upper arm was placed on a table in a horizontal state. The angle of the forearm was measured with a modified finger goniometer, which intrinsically consists of a protractor, string, and plumb. ${ }^{(9)}$

\section{Results and Discussion}

The relationship between the sensor resistance and stretching, based on the results of the static stretching test, is shown in Fig. 5(a). The overall sensitivity was found to be -1.98 $\mathrm{k} \Omega / \mathrm{mm}$. The standard deviations were 2.85 and $1.16 \mathrm{k} \Omega$ at the first and last positions, respectively. Even though the linearity was insufficient, it could be calibrated using curve fitting.

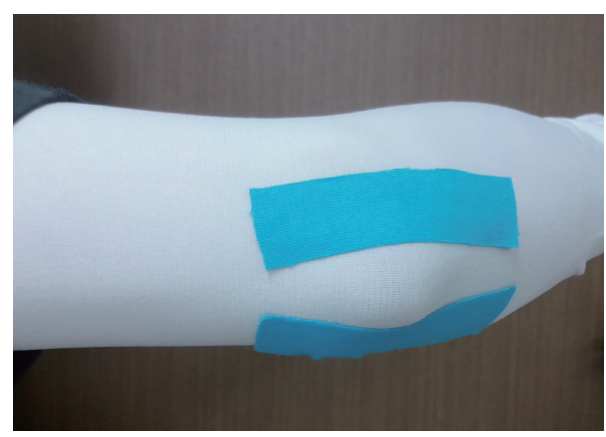

Fig. 4. (Color online) Two sensors attached on the elbow.

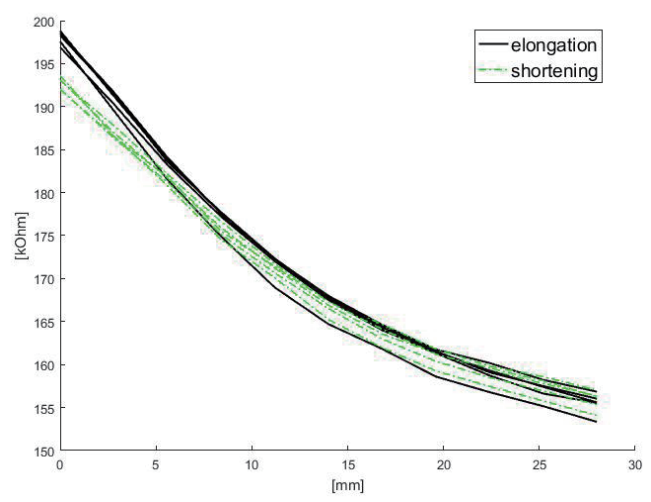

(a)

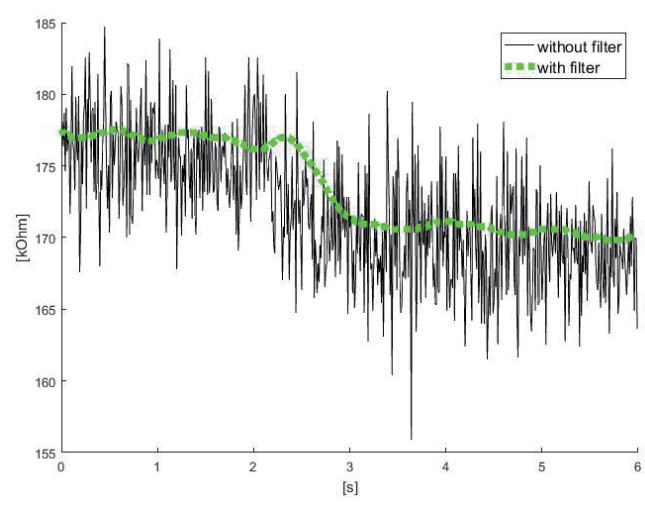

(b)

Fig. 5. (Color online) (a) Static characteristics (black, elongation; green, shortening) and (b) dynamic characteristics (black, without filter; green, with filter). 


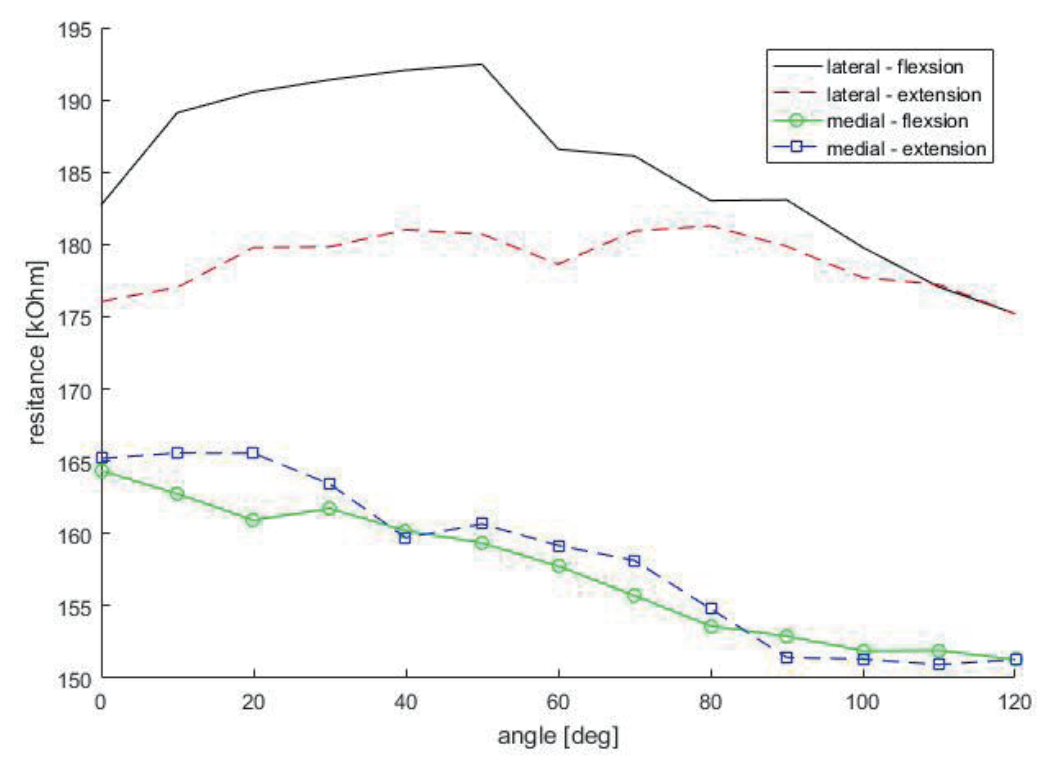

Fig. 6. (Color online) Sensor characteristics for the elbow angle.

The dynamic stretching test shows a reasonable sensor response [Fig. 5(b)]. It also confirms the necessity of low-pass filtering. Without the filter, the standard deviations were 3.32 and 3.41 $\mathrm{k} \Omega$, before and after the stretching for $2 \mathrm{~s}$. With the filter, they decreased to 0.30 and $0.37 \mathrm{k} \Omega$.

The characteristics of the two sensors attached on the elbow did not show satisfactory repeatability over the six cycles. The reason for this was that the sensors moved during the flexion and extension cycle. The attachment of the sensor on the stretchable arm sleeve was good with the adhesive tape. However, the attachment of the sleeve on the skin was not sufficiently firm.

Despite the issues mentioned above, there were several meaningful findings. Figure 6 shows a cycle of flexion and extension. Firstly, the sensor attached on the lateral side showed an undesired increasing pattern between 0 and $50^{\circ}$, and a reasonable decreasing pattern above $50^{\circ}$. The undesired increasing pattern resulted from the fact that the sensors were in a collapsed state, i.e., without any tension. The reason for this is the sleeve-skin contact issue mentioned above. Secondly, the lateral side sensor above $50^{\circ}$ and the medial sensor for the full range showed reasonable sensitivity. Thirdly, the lateral sensor showed a complementary pattern with the medial sensor, and this pattern could be utilized within a sensor fusion context. Finally, the sensor textile and adhesive tape did not provide sufficient stretching elongation for the stretching of the skin along the coronoid process.

\section{Conclusions}

In this study, we proposed a human joint angle estimation method with e-textile strainresistance sensors. The stretching shape of the skin, on which the sensor is attached, was visually analyzed in terms of the stretching capability of the sensor and the actual stretching 
length of the skin, which was the main aim of this study. The overall system platform used to obtain and process the sensor data was described in detail. The static and dynamic characteristics of the sensor were tested with a linear actuator. The configuration of the sensor array and the attachment location were designed considering the stretching shape of the skin. The proposed system was implemented and applied to the elbow joint of a healthy subject. It was found that the sensors should be attached to the skin properly, especially when the skin is not stretched. To address this issue, a simple model of an elbow would be more appropriate for this application. A simple elbow model, such as a hinge with a uniform cylinder, will be adopted in a future study. It is necessary to consider the stretching capability of the sensor to accommodate the actual stretching of the skin. The two sensors attached on the lateral and medial sides provide complementary information, which can be utilized in a sensor fusion context. For instance, the undesired increasing pattern of the lateral sensor could be utilized to compensate the non-increasing pattern of the medial sensor. Even though only the elbow-joint estimation was attempted in this study, estimations could be carried out for other human joints such as the shoulder and wrist. The estimation of human joint angles is expected to be used for various applications such as designing natural user interfaces and upper limb rehabilitation.

\section{Acknowledgments}

This research was supported by the Basic Science Research Program through the National Research Foundation of Korea (NRF) funded by the Ministry of Education (NRF2017R1D1A1B03029739).

\section{References}

1 M. Stoppa and A. Chiolerio: Sensors 14 (2014) 7.

2 H. C. Yu, C. L. Cheng, P. H. Wu, and S. J. Li: Sens. Mater. 29 (2017) 1.

3 W. Xu, Z. Li, M. C. Huang, N. Amini, and M. Sarrafzadeh: Proc. 2011 Body Sensor Networks Conf. (IEEE, 2011) 194.

4 C. Mattmann, O. Amft, H. Harms, G. Troster, and F. Clemens: Proc. 2007 11th IEEE Int. Symp. (IEEE, 2007) 29.

5 T. W. Shyr, J. W. Shie, C. H. Jiang, and J. J. Li: Sensors 14 (2014) 3.

6 A. Tognetti, F. Lorussi, G. Dalle Mura, N. Carbonaro, M. Pacelli, R. Paradiso, and D. De Rossi: J. Neuroeng. Rehabil. 11 (2014) 1.

7 N. Carbonaro, G. Dalle Mura, F. Lorussi, R. Paradiso, D. De Rossi, and A. Tognetti: IEEE J. Biomed. Health. Inf. 18 (2014) 6.

8 M. Rasouli, R. Ghosh, W. W. Lee, N. V. Thakor, and S. Kukreja: Proc. 2015 37th Annu. Int. Conf. IEEE Engineering in Medicine and Biology Society (IEEE, 2015) 4828.

9 M. Szekeres, J. C. MacDermid, and J. Rooney: J. Hand Ther. 28 (2015) 4. 